\title{
PERMANENTLY WEAK AMENABILITY OF REES SEMIGROUP ALGEBRAS
}

\author{
HASAN HOSSEINZADEH ${ }^{1}$ AND ALI JABBARI ${ }^{2}$ \\ ${ }^{1}$ Department of Mathematics, Ardabil Branch, Islamic Azad University, Ardabil, Iran \\ ${ }^{2}$ Department of Mathematics, Payame Noor University, Tehran, Iran \\ ${ }^{*}$ Corresponding author: jabbari_al@yahoo.com
}

\begin{abstract}
In this paper, we consider $n$-weak amenability of full matrix algebras and we prove that the Rees semigroup algebra is permanently weakly amenable.
\end{abstract}

\section{INTRODUCTION}

Let $A$ be a Banach algebra, and let $X$ be a Banach $A$-bimodule. Then a linear map $D: A \longrightarrow X$ is a derivation if

$$
D(a b)=a \cdot D(b)+D(a) \cdot b
$$

for every $a, b \in A$. Let $x \in X$, and set $\delta_{x}(a)=a \cdot x-x \cdot a$ for every $a \in A$. Then $\delta_{x}$ is a derivation; these derivations are inner derivations. The space of continuous derivations from $A$ into $X$ is denoted by $\mathcal{Z}^{1}(A, X)$, and the subspace consisting of the inner derivations is $\mathcal{N}^{1}(A, X)$; the first cohomology group of $A$ with coefficients in $X$ is $\mathcal{H}^{1}(A, X)=\mathcal{Z}^{1}(A, X) / \mathcal{N}^{1}(A, X)$.

A Banach algebra $A$ is weakly amenable if $\mathcal{H}^{1}\left(A, A^{*}\right)=\{0\}$. For example, the group algebra $L^{1}(G)$ is weak amenable for each locally compact group $G[7]$.

Let $k \in \mathbb{N}$; a Banach algebra $A$ is called $k$-weakly amenable if $\mathcal{H}^{1}\left(A, A^{(k)}\right)=\{0\}$. Dales, Ghahramani and Grønbæk brought the concept of $k$-weak amenability of Banach algebras [5]. A Banach algebra $A$ is called

Received $23^{\text {rd }}$ September, 2017; accepted $7^{\text {th }}$ December, 2017; published $3^{\text {rd }}$ January, 2018.

2010 Mathematics Subject Classification. Primary 43A07, Secondary 46H25.

Key words and phrases. amenability; inverse semigroup; Rees semigroup; weak amenability.

(C) 2018 Authors retain the copyrights of their papers, and all open access articles are distributed under the terms of the Creative Commons Attribution License. 
permanently weakly amenable if $H^{1}\left(A, A^{(k)}\right)=\{0\}$, for each $k \in \mathbb{N}$. In [5], authors showed that for a locally compact group $G, L^{1}(G)$ is $n$-weakly amenable for all odd numbers $n$, but for even case this was open. This open problem solved in [4] and a new prove introduced by Zhang [9].

The above mentioned problem open for semigroups and semigroup algebras. For Rees semi group algebras, Mewomo [8], proved that these algebras are $(2 k+1)$-weakly amenable, in this paper, we investigate permanent weak amenability of $n \times n$ matrix Banach algebras. Finally, we prove that the Rees semigroup algebras are permanently weak amenable.

\section{Characterization of Derivations}

Consider the algebra $M_{n}$ of $n \times n$ matrices. Let $A$ be a Banach algebra. The Banach algebra $M_{n}(A)$ is the collection of $n \times n$ matrices with components in $A$. We identify the dual of $M_{n}(A)$ with $M_{n}\left(A^{*}\right)$ and we have

$$
(a \cdot \Lambda)_{i j}=\sum_{s=1}^{n} a_{j s} \cdot \lambda_{i s}, \quad(\Lambda \cdot a)_{i j}=\sum_{s=1}^{n} \lambda_{s j} \cdot a_{s i},
$$

for each $a=\left(a_{i j}\right) \in M_{n}(A)$ and $\Lambda=\left(\lambda_{i j}\right) \in M_{n}\left(A^{*}\right)$.

Derivations from $M_{n}(A)$ into $M_{n}\left(A^{*}\right)$ is studied in [1]. Set $E_{i j}$ which it is a $n \times n$ matrix, such that whose $(i, j)^{t h}$ entry is 1 and other entries are 0 . For each $a \in A$, the matrix $a \otimes E_{i j}$ is a matrix that whose $(i, j)^{t h}$ entry is $a$ and others entries are 0.

Lemma 2.1. Let $A$ be a Banach algebra and let $D: A \longrightarrow A^{*}$ be a continuous derivation, then $D$ induces a continuous derivation $\mathfrak{D}: M_{n}(A) \longrightarrow M_{n}\left(A^{*}\right)$. Moreover, if $\mathfrak{D}$ is an inner derivation, then $D$ is inner derivation.

Proof. Define $\mathfrak{D}: M_{n}(A) \longrightarrow M_{n}\left(A^{*}\right)$ by $\mathfrak{D}\left((a)_{i j}\right)=\left(D\left(a_{i j}\right)\right)$ or $\mathfrak{D}\left((a)_{i j}\right)=\left(D\left(a_{j i}\right)\right)$. Clearly, continuity of $D$ implies continuity of $\mathfrak{D}$. Similar to argumentation in [6, pp. 17], we have $\mathfrak{D}(a b)=a \cdot \mathfrak{D}(b)+\mathfrak{D}(a) \cdot b$ for every $a, b \in M_{n}(A)$. Thus, $\mathfrak{D}$ is a module derivation. As well as, if $\mathfrak{D}$ is inner, by a similar method in proof of Theorem 2.7 of [6], $D$ is inner.

By $(2.1)$,

$$
\begin{aligned}
\left\langle\lambda \otimes E_{k l},\left(\Lambda_{i j}\right) \cdot\left(a_{i j}\right)\right\rangle & =\left\langle\left(a_{i j}\right) \cdot\left(\lambda \otimes E_{k l}\right),\left(\Lambda_{i j}\right)\right\rangle \\
& =\left\langle\sum_{s=1}^{n}\left(a_{s l} \cdot \lambda \otimes E_{k l}\right),\left(\Lambda_{i j}\right)\right\rangle=\sum_{s=1}^{n}\left\langle a_{s l} \cdot \lambda, \Lambda_{k s}\right\rangle \\
& =\left\langle\lambda, \sum_{s=1}^{n} \Lambda_{k s} \cdot a_{s l}\right\rangle,
\end{aligned}
$$

for each $\lambda \in A^{*},\left(\Lambda_{i j}\right) \in M_{n}\left(A^{* *}\right),\left(a_{i j}\right) \in M_{n}(A)$ and $0 \leq k, l \leq n$. Hence, (2.2) implies that

$$
\left(\left(\Lambda_{i j}\right) \cdot\left(a_{i j}\right)\right)_{k l}=\sum_{s=1}^{n} \Lambda_{k s} \cdot a_{s l}
$$


for each $\left(\Lambda_{i j}\right) \in M_{n}\left(A^{* *}\right),\left(a_{i j}\right) \in M_{n}(A)$ and $0 \leq k, l \leq n$. Similarly

$$
\left(\left(a_{i j}\right) \cdot\left(\Lambda_{i j}\right)\right)_{k l}=\sum_{s=1}^{n} a_{k s} \cdot \Lambda_{s l}
$$

for each $\left(\Lambda_{i j}\right) \in M_{n}\left(A^{* *}\right),\left(a_{i j}\right) \in M_{n}(\mathcal{A})$ and $0 \leq k, l \leq n$.

By induction on $m$, for each $\left(a_{i j}\right) \in M_{n}(\mathcal{A})$ and $\left(\lambda_{i j}\right) \in M_{n}\left(A^{(m)}\right)$ we have

$$
\left(\left(\lambda_{i j}\right) \cdot\left(a_{i j}\right)\right)_{k l}=\sum_{s=1}^{n} \lambda_{s l} \cdot a_{s k}, \quad\left(\left(a_{i j}\right) \cdot\left(\lambda_{i j}\right)\right)_{k l}=\sum_{s=1}^{n} a_{l s} \cdot \lambda_{k s},
$$

when $m$ is odd and in the case where $m$ is even, we have the following actions:

$$
\left(\left(\lambda_{i j}\right) \cdot\left(a_{i j}\right)\right)_{k l}=\sum_{s=1}^{n} \lambda_{k s} \cdot a_{s l}, \quad\left(\left(a_{i j}\right) \cdot\left(\lambda_{i j}\right)\right)_{k l}=\sum_{s=1}^{n} a_{k s} \cdot \lambda_{s l} .
$$

Now; we are ready to prove the following Lemma that plays an important role in our main results.

Lemma 2.2. Let $A$ be a unital Banach algebra. Then every derivation from $M_{n}(A)$ into $M_{n}\left(A^{(m)}\right)\left(A^{(m)}\right.$ is the $m$-th dual of $A$ ) is the sum of an inner derivation and a derivation induced by a derivation from $A$ into $A^{(m)}$.

Proof. Let $e_{A}$ be the identity element of $A$. Suppose that $\mathfrak{D}: M_{n}(A) \longrightarrow M_{n}\left(A^{(m)}\right)$ is a continuous derivation. For each $i, j$ and $k, l$, define $D_{i j}^{k l}: A \longrightarrow A^{(m)}$ by $D_{i j}^{k l}(a):=\left(\mathfrak{D}\left(a \otimes E_{i j}\right)\right)_{k l}$, for each $a \in A$. Clearly, $D_{i j}^{k l}$ is linear. We prove this Lemma in two cases.

Case 1. Let $m$ be an odd positive number. For every $a, b \in A$ and each $1 \leq t \leq n$, we have

$$
\begin{aligned}
\left(\left[\mathfrak{D}\left(a \otimes E_{i t}\right)\right] \cdot\left(b \otimes E_{t j}\right)\right)_{k l} & =\sum_{s=1}^{n}\left(\mathfrak{D}\left(a \otimes E_{i t}\right)\right)_{s l} \cdot\left(b \otimes E_{t j}\right)_{s k} \\
& =\sum_{s=1}^{n} D_{i t}^{s l}(a) \cdot b \delta_{t s} \delta_{j k}=D_{i t}^{t l}(a) \cdot b \delta_{j k}
\end{aligned}
$$

and

$$
\begin{aligned}
\left(\left(a \otimes E_{i t}\right) \cdot\left[\mathfrak{D}\left(b \otimes E_{t j}\right)\right]\right)_{k l} & =\sum_{s=1}^{n}\left(a \otimes E_{i t}\right)_{l s} \cdot\left(\mathfrak{D}\left(b \otimes E_{t j}\right)\right)_{k s} \\
& =\sum_{s=1}^{n} a \delta_{i l} \delta_{t s} \cdot D_{t j}^{k s}(b)=a \delta_{i l} \cdot D_{t j}^{k t}(b)
\end{aligned}
$$

where $\delta$ is the Kronecker's delta. Then

$$
D_{i j}^{k l}(a b)=a \delta_{i l} \cdot D_{t j}^{k t}(b)+D_{i t}^{t l}(a) \cdot b \delta_{j k}
$$

Thus, $D_{i i}^{i i}$ is a derivation from $A$ into $A^{(m)}$. From (2.5) and (2.7), the following statements hold

$$
D_{i j}^{j l}(a)=D_{i i}^{i l}\left(e_{A}\right) \cdot a \quad(i \neq l), \quad D_{i j}^{k i}(a)=a \cdot D_{j j}^{k j}\left(e_{A}\right) \quad(j \neq k)
$$


and again by (2.7) and for $1 \leq i, j, l \leq n$, we have

$$
\begin{aligned}
D_{j j}^{j j}(a) & =D_{j i}^{i j}\left(e_{A}\right) \cdot a+D_{i j}^{j i}(a)=D_{j i}^{i j}\left(e_{A}\right) \cdot a+D_{i l}^{l i}\left(e_{A}\right) \cdot a+D_{l j}^{j l}(a) \\
& =D_{j i}^{i j}\left(e_{A}\right) \cdot a+D_{i l}^{l i}\left(e_{A}\right) \cdot a+D_{l l}^{l l}(a)+a \cdot D_{l j}^{j l}\left(e_{A}\right),
\end{aligned}
$$

and

$$
D_{j i}^{i j}(a)=a \cdot D_{j i}^{i j}\left(e_{A}\right)+D_{j j}^{j j}(a)
$$

Hence $D_{j i}^{i j}\left(e_{A}\right)=-D_{i j}^{j i}\left(e_{A}\right)$ for every $1 \leq i, j \leq n$, and consequently by $(2.9)$, the following relation holds

$$
D_{i j}^{j i}(a)=D_{i l}^{l i}\left(e_{A}\right) \cdot a-a \cdot D_{j l}^{l j}\left(e_{A}\right)+D_{l l}^{l l}(a)
$$

Together with (2.9) and (2.10) we have

$$
D_{i j}^{j i}(a)=D_{j i}^{i j}(a)-D_{j i}^{i j}\left(e_{A}\right) \cdot a-a \cdot D_{j i}^{i j}\left(e_{A}\right),
$$

for every $a \in A$. By (2.7) and (2.10) the following equality holds

$$
\begin{aligned}
D_{k l}^{i j}(a) & =D_{k i}^{i j}\left(e_{A}\right) \cdot a+D_{i l}^{i i}(a)=D_{k i}^{i j}\left(e_{A}\right) \cdot a+D_{i j}^{j i}\left(e_{A}\right) \cdot a+D_{j l}^{i j}(a) \\
& =D_{k i}^{i j}\left(e_{A}\right) \cdot a+D_{i j}^{j i}\left(e_{A}\right) \cdot a+a \cdot D_{j l}^{i j}\left(e_{A}\right)+D_{j j}^{j j}(a) \\
& =D_{k i}^{i j}\left(e_{A}\right) \cdot a+a \cdot D_{j l}^{i j}\left(e_{A}\right)-D_{j i}^{i j}\left(e_{A}\right) \cdot a-a \cdot D_{j i}^{i j}\left(e_{A}\right)+D_{j i}^{i j}(a),
\end{aligned}
$$

for every $a \in A$. Then by (2.8), (2.12) and (2.13), we have

$$
\begin{aligned}
\left(\mathfrak{D}\left(a_{r s}\right)\right)_{i j}= & \sum_{k, l=1}^{n} D_{k l}^{i j}\left(a_{k l}\right)=\sum_{k=1}^{n} D_{k i}^{i j}\left(e_{A}\right) \cdot a_{k i}+\sum_{l=1}^{n} D_{i l}^{i i}\left(a_{i l}\right) \\
= & \sum_{k=1}^{n} D_{k i}^{i j}\left(e_{A}\right) \cdot a_{k i}+\sum_{l=1}^{n} a_{j l} \cdot D_{j l}^{i j}\left(e_{A}\right) \\
& -D_{j i}^{i j}\left(e_{A}\right) \cdot a_{j i}-a_{j i} \cdot D_{j i}^{i j}\left(e_{A}\right)+D_{j i}^{i j}\left(a_{j i}\right) \\
= & \sum_{k=1}^{n} D_{k k}^{k j}\left(e_{A}\right) \cdot a_{k i}+\sum_{k=1}^{n} a_{j k} \cdot D_{k k}^{i k}\left(e_{A}\right)+D_{i j}^{j i}\left(a_{j i}\right),
\end{aligned}
$$

for every $\left(a_{r s}\right) \in M_{n}(\mathcal{A})$. As well as,

$$
\left(\mathfrak{D}\left(E_{k k} E_{i i}\right)\right)_{i k}=\sum_{k=1}^{n} D_{k k}^{s k}\left(e_{A}\right) \delta_{s i}+\sum_{k=1}^{n} \delta_{k s} D_{i i}^{i s}\left(e_{A}\right)=D_{k k}^{i k}\left(e_{A}\right)+D_{i i}^{i k}\left(e_{A}\right)=0 .
$$

This shows that $D_{k k}^{i k}\left(e_{A}\right)=-D_{i i}^{i k}\left(e_{A}\right)$. Now; for every $1 \leq k, j \leq n$ define $D_{k j}=D_{k k}^{k j}$. By the above obtained results we have

$$
\begin{aligned}
\left(\mathfrak{D}\left(a_{r s}\right)\right)_{i j} & =\sum_{k=1}^{n} D_{k j}\left(e_{A}\right) \cdot a_{k i}-\sum_{k=1}^{n} a_{j k} \cdot D_{i k}\left(e_{A}\right)+D_{i j}^{j i}\left(a_{j i}\right) \\
& =\left(\left(D_{r s}\left(e_{A}\right)\right) \cdot\left(a_{r s}\right)-\left(a_{r s}\right) \cdot\left(D_{r s}\left(e_{A}\right)\right)\right)_{i j}+D_{i j}^{j i}\left(a_{j i}\right) .
\end{aligned}
$$


Set

$$
\mathcal{D}\left(e_{A}\right)=\left[\begin{array}{ccc}
D_{1 l}^{l 1}\left(e_{A}\right) & \ldots & 0 \\
\vdots & D_{2 l}^{l 2}\left(e_{A}\right) & \vdots \\
0 & \ldots & D_{n l}^{l n}\left(e_{A}\right)
\end{array}\right]_{n \times n} .
$$

Then by (2.11) and (2.15) we have

$$
\begin{aligned}
\mathfrak{D}\left(\left(a_{r s}\right)\right)= & \left(D_{i j}\left(e_{A}\right)+\mathcal{D}\left(e_{A}\right)\right) \cdot\left(a_{i j}\right)-\left(a_{i j}\right) \cdot\left(D_{i j}\left(e_{A}\right)+\mathcal{D}\left(e_{A}\right)\right) \\
& +\left(D_{l l}^{l l}\left(a_{i j}\right)\right),
\end{aligned}
$$

where $\left(D_{l l}^{l l}\left(a_{i j}\right)\right)$ is a diagonal matrix.

Case 2. Now; let $m$ be an even positive number. Then by (2.6) we have

$$
\begin{aligned}
\left(\left[\mathfrak{D}\left(a \otimes E_{i t}\right)\right] \cdot\left(b \otimes E_{t j}\right)\right)_{k l} & =\sum_{s=1}^{n}\left(\mathfrak{D}\left(a \otimes E_{i t}\right)\right)_{k s} \cdot\left(b \otimes E_{t j}\right)_{s l} \\
& =\sum_{s=1}^{n} D_{i t}^{k s}(a) \cdot b \delta_{t s} \delta_{j l}=D_{i t}^{k t}(a) \cdot b \delta_{j l}
\end{aligned}
$$

and

$$
\begin{aligned}
\left(\left(a \otimes E_{i t}\right) \cdot\left[\mathfrak{D}\left(b \otimes E_{t j}\right)\right]\right)_{k l} & =\sum_{s=1}^{n}\left(a \otimes E_{i t}\right)_{k s} \cdot\left(\mathfrak{D}\left(b \otimes E_{t j}\right)\right)_{s l} \\
& =\sum_{s=1}^{n} a \delta_{i k} \delta_{t s} \cdot D_{t j}^{s l}(b)=a \delta_{i k} \cdot D_{t j}^{t l}(b),
\end{aligned}
$$

for every $a, b \in A$. Then

$$
D_{i j}^{k l}(a b)=a \delta_{i k} \cdot D_{t j}^{t l}(b)+D_{i t}^{k t}(a) \cdot b \delta_{j l} .
$$

Thus, $D_{i i}^{i i}$ is a derivation from $A$ into $A^{(m)}$. By (2.6) and (2.16), the following equalities hold

$$
D_{i j}^{k j}(a)=D_{i i}^{k i}\left(e_{A}\right) \cdot a \quad(k \neq i), \quad D_{i j}^{i l}(a)=a \cdot D_{j j}^{j l}\left(e_{A}\right) \quad(j \neq l),
$$

and for $1 \leq i, j, l \leq n,(2.16)$ follows

$$
\begin{aligned}
D_{i i}^{i i}(a) & =D_{j i}^{j i}(a)+D_{i j}^{i j}\left(e_{A}\right) \cdot a=D_{i j}^{i j}\left(e_{A}\right) \cdot a+D_{j l}^{j l}\left(e_{A}\right) \cdot a+D_{l i}^{l i}(a) \\
& =D_{i j}^{i j}\left(e_{A}\right) \cdot a+D_{j l}^{j l}\left(e_{A}\right) \cdot a+D_{l l}^{l l}(a)+a \cdot D_{l i}^{l i}\left(e_{A}\right),
\end{aligned}
$$

and

$$
D_{j i}^{j i}(a)=D_{i i}^{i i}(a)+D_{j i}^{j i}\left(e_{A}\right) \cdot a,
$$

for every $a \in A$. Therefore $D_{i j}^{i j}\left(e_{A}\right)=-D_{j i}^{j i}\left(e_{A}\right)$, for every $1 \leq i, j \leq n$. Then (2.18) implies that

$$
D_{j i}^{j i}(a)=D_{j l}^{j l}\left(e_{A}\right) \cdot a-a \cdot D_{i l}^{i l}\left(e_{A}\right)+D_{l l}^{l l}(a) .
$$

As well as,

$$
D_{k l}^{i j}(a)=D_{k j}^{i j}\left(e_{A}\right) \cdot a+D_{i l}^{j j}(a)=D_{k j}^{i j}\left(e_{A}\right) \cdot a+a \cdot D_{i l}^{i j}\left(e_{A}\right)+D_{j i}^{j i}(a)
$$


for every $a \in A$. By using the relations (2.17) and (2.21), for every $\left(a_{r s}\right) \in M_{n}(A)$, we have

$$
\begin{aligned}
\left(\mathfrak{D}\left(a_{r s}\right)\right)_{i j} & =\sum_{k, l=1}^{n} D_{k l}^{i j}\left(a_{k l}\right)=\sum_{l=1}^{n} D_{k j}^{i j}\left(e_{A}\right) \cdot a_{k j}+\sum_{k=1}^{n} D_{i l}^{j j}\left(a_{i l}\right) \\
& =\sum_{k=1}^{n} D_{k j}^{i j}\left(e_{A}\right) \cdot a_{k j}+\sum_{k=1}^{n} a_{i l} \cdot D_{i l}^{i j}\left(e_{A}\right)+D_{j i}^{j i}\left(a_{j i}\right) \\
& =\sum_{k=1}^{n} D_{k k}^{i k}\left(e_{A}\right) \cdot a_{k j}+\sum_{k=1}^{n} a_{i k} \cdot D_{k k}^{k j}\left(e_{A}\right)+D_{j i}^{j i}\left(a_{j i}\right) .
\end{aligned}
$$

Since

$$
\left(\mathfrak{D}\left(E_{k k} E_{i i}\right)\right)_{i k}=\sum_{k=1}^{n} D_{k k}^{k s}\left(e_{A}\right) \delta_{i s}+\sum_{k=1}^{n} \delta_{i s} D_{i i}^{s k}\left(e_{A}\right)=D_{k k}^{k i}\left(e_{A}\right)+D_{i i}^{i k}\left(e_{A}\right)=0,
$$

$D_{k k}^{k i}\left(e_{A}\right)=-D_{i i}^{i k}\left(e_{A}\right)$. Now; define $D_{k j}=D_{k k}^{k j}$ for every $1 \leq j, k \leq n$. Then by the above obtained results we have

$$
\begin{aligned}
\left(\mathfrak{D}\left(a_{r s}\right)\right)_{i j} & =\sum_{k=1}^{n} D_{i k}\left(e_{A}\right) \cdot a_{k j}-\sum_{k=1}^{n} a_{i k} \cdot D_{j k}\left(e_{A}\right)+D_{j i}^{j i}\left(a_{j i}\right) \\
& =\left(\left(D_{r s}\left(e_{A}\right)\right) \cdot\left(a_{r s}\right)-\left(a_{r s}\right) \cdot\left(D_{r s}\left(e_{A}\right)\right)\right)_{i j}+D_{j i}^{j i}\left(a_{j i}\right)
\end{aligned}
$$

Similar to Case 1, set

$$
\mathcal{D}\left(e_{A}\right)=\left[\begin{array}{ccc}
D_{1 l}^{1 l}\left(e_{A}\right) & \ldots & 0 \\
\vdots & D_{2 l}^{2 l}\left(e_{A}\right) & \vdots \\
0 & \ldots & D_{n l}^{n l}\left(e_{A}\right)
\end{array}\right]_{n \times n} .
$$

Now; by applying (2.20) and (2.24) the following holds

$$
\begin{aligned}
\mathfrak{D}\left(\left(a_{r s}\right)\right)= & \left(D_{i j}\left(e_{A}\right)+\mathcal{D}\left(e_{A}\right)\right) \cdot\left(a_{i j}\right)-\left(a_{i j}\right) \cdot\left(D_{i j}\left(e_{A}\right)+\mathcal{D}\left(e_{A}\right)\right) \\
& +\left(D_{l l}^{l l}\left(a_{i j}\right)\right) .
\end{aligned}
$$

Hence proof is complete.

Weak amenability and $(2 k+1)$-weak amenability of $M_{n}(A)$ considered in [3, 8]. Now; by above Lemma we have the following result:

Theorem 2.1. Let $A$ be a unital Banach algebra. Then $A$ is permanently weakly amenable if and only if $M_{n}(A)$ is permanently weakly amenable.

Proof. Let $M_{n}(A)$ be permanently weakly amenable and let $D: A \longrightarrow A^{(k)}$ be a continuous derivation, $k \in \mathbb{N}$. Then by Lemma 2.1, $D$ induces a continuous derivation $\mathfrak{D}: M_{n}(A) \longrightarrow M_{n}\left(A^{(k)}\right)$. Hence, by our assumption $\mathfrak{D}$ is inner and Lemma 2.1, implies that $D$ is inner.

Conversely, suppose that $A$ is permanently weakly amenable. Let $\mathfrak{D}: M_{n}(A) \longrightarrow M_{n}\left(A^{(k)}\right)$ be a continuous module derivation, $k \in \mathbb{N}$. Then by Lemma 2.2 , it is equal to the sum of an inner derivation and a 
derivation induced by a derivation from $A$ into $A^{(k)}$. Since $A$ is permanently weakly module, $\mathfrak{D}$ is equal to sum of two inner derivations. Thereby, $M_{n}(A)$ is permanently weakly module amenable.

Example 2.1. Let $G$ be a discrete group. Then by [4], [5] and Theorem 2.1, $M_{n}\left(\ell^{1}(G)\right)$ is permanently weakly amenable.

Example 2.2. Let $A$ be a unital $C^{*}$-algebra. Then $M_{n}(A)$ is permanently weakly amenable.

\section{Rees semigroup algebras}

Let $G$ be a group, and $m, n \in \mathbb{N}$; the zero adjoined to $G$ is $o$. A Rees semigroup has the form $S=$ $\mathcal{M}(G, P, m, n)$; here $P=\left(a_{i j}\right) \in M_{n, m}(G)$ is the collection of $n \times m$ matrices with components in $G$. For $x \in G, 1 \leq i \leq m$ and $1 \leq j \leq n$, let $(x)_{i j}$ be the element of $M_{m, n}\left(G^{o}\right)$ with $x$ in the $(i, j)$-th place and $o$ elsewhere. As a set, $S$ consists of the collection of all these matrices $(x)_{i j}$. Multiplication in $S$ is given by the formula

$$
(x)_{i j}(y)_{k l}=\left(x a_{j k} y\right)_{i l} \quad(x, y \in G, 1 \leq i, k \leq m, 1 \leq j, l \leq n)
$$

It is known that $S$ is a semigroup. Now; consider the semigroup $\mathcal{M}^{o}(G, P, m, n)$, where the elements of this semigroup are those of $\mathcal{M}(G, P, m, n)$, together with the element $o$, identified with the matrix that has $o$ in each place (so that $o$ is the zero of $\mathcal{M}^{o}(G, P, m, n)$ ), and the components of $P$ are belong to $G^{o}$. The matrix $P$ is called the sandwich matrix in each case. The semigroup $\mathcal{M}^{o}(G, P, m, n)$ is a Rees matrix semigroup with a zero over $G$. We write $\mathcal{M}^{o}(G, P, n)$ for $\mathcal{M}^{o}(G, P, n, n)$ in the case where $m=n$. As well as, $P$ is called regular if every row and column contains at least one entry in $G$. The semigroup $\mathcal{M}^{o}(G, P, m, n)$ is regular as a semigroup if and only if the sandwich matrix $P$ is regular.

According to [6] we have the following equalities as Banach spaces

$$
\ell^{1}(S)=\mathcal{M}^{o}\left(\ell^{1}(G), P, m, n\right)=\mathcal{M}\left(\ell^{1}(G), P, m, n\right) \oplus \mathbb{C} \delta_{0}
$$

Bowling and Duncan proved that for any Rees semigroup $S, \ell^{1}(S)$ is weakly amenable [3, Theorem 2.5] and after them Mewomo in [8], proved that $\ell^{1}(S)$ is $(2 k+1)$-weakly amenable where $S=\mathcal{M}^{o}(G, P, n)$, for $k, n \in \mathbb{N}$. Now; we are completing them works as follows:

Theorem 3.1. Let $S=\mathcal{M}^{o}(G, P, n), n \in \mathbb{N}$. Then $\ell^{1}(S)$ is permanently weakly amenable.

Proof. It is sufficient we show that $\ell^{1}(S)$ is $(2 k)$-weakly amenable, for $k \in \mathbb{N}$. For any locally compact group $G, \ell^{1}(G)$ is permanently weakly amenable ( $[4$, pp. 3179] and [5, Theorem 4.1]). Theorem 2.1 implies that $M_{n}\left(\ell^{1}(G)\right)$ is $(2 k)$-weakly amenable. Since $M_{n}\left(\ell^{1}(G)\right)=\ell^{1}(S), \ell^{1}(S)$ is $(2 k)$-weakly amenable.

Let $S$ be a semigroup. The weak amenability of $\ell^{1}(S)$ is considered by Blackmore in [2]. He proved that $\ell^{1}(S)$ to be weakly amenable whenever $S$ is completely regular, in the sense that, for each $s \in S$, there exists 
$t \in S$ with $s t s=s$ and $s t=t s$. Suppose that $S$ has a zero $o$. Then $S$ is o-simple if $S_{[2]} \neq\{o\}$ and the only ideals in $S$ are $\{o\}$ and $S$. The semigroup $S$ is called completely o-simple if it is $o$-simple and contains a primitive idempotent.

Corollary 3.1. Let $S$ be an infinite, completely o-simple semigroup with finitely many idempotents. Then $\ell^{1}(S)$ is permanently weakly amenable.

Proof. By Corollary 4.2 of [8], it suffices to show that $\ell^{1}(S)$ is $(2 k)$-weakly amenable, for $k \in \mathbb{N}$. The semigroup $S$ is isomorphic as a semigroup to a regular Rees matrix semigroup with a zero $\mathcal{M}^{o}(G, P, n)$, $n \in \mathbb{N}[6$, Theorem 3.13]. Now; apply Theorem 3.1.

Acknowledgment. The authors wish to thank Professor Yong Zhang for pointing out the reference [9] in response to an earlier version of this article. This work is supported by a grant from the Ardabil Branch, Islamic Azad University. Thus; the first author would like to express his deep gratitude to the Ardabil Branch, Islamic Azad University, for financial supports.

\section{REFERENCES}

[1] R. Alizadeh and G. Esslamzadeh, The structure of derivations from a full matrix algebra into its dual, Iranian J. Sci. Tec, Trans. A, 32(2008), 61-64.

[2] T. D. Blackmore, Weak amenability of discrete semigroup algebras, Semigroup Forum, 55(1997), $196-205$.

[3] S. Bowling and J. Duncan, First order cohomology of Banach semigroup algebras, Semigroup Forum, 56(1998), $130-145$.

[4] Y. Choi, F. Ghahramani, Y. Zhang, Approximate and pseudo-amenability of various classes of Banach algebras, J. Funct. Anal., 256(2009), 3158-3191.

[5] H. G. Dales, F. Ghahramani and N. Grønbæk, Derivatios into iterated duals of Banach algebras, Studia Math., 128(1)(1998), $19-54$.

[6] H. G. Dales, A. T-M. Lau and D. Strauss, Banach algebras on semigroups and their compactifications, Memoirs Amer. Math. Soc., 205, American Mathematical Society, Providence, 2010.

[7] B. E. Johnson, Weak amenability of group algebras, Bull. London Math. Soc., 23(1991), 281-284.

[8] O. T. Mewomo, On n-weak amenability of Rees semigroup algebras, Proc. Indian Acad. Sci., 118(4)(2008), 547-555.

[9] Y. Zhang, 2m-Weak amenability of group algebras, J. Math. Anal. Appl., 396(2012), 412-416. 